\title{
Mixed thermal convection: fundamental issues and analysis of the planar case
}

\author{
JACQUES PADET $^{1}$, RENATO M. COTTA $^{2}$, EMILIA C. MLADIN $^{3}$ and COLETTE PADET ${ }^{1}$ \\ ${ }^{1}$ Université de Reims, GRESPI - Thermomécanique, Faculté des Sciences, B.P. 1039, 51687 Reims, France \\ ${ }^{2}$ Laboratório de Transmissão e Tecnologia do Calor/LTTC, Programa de Engenharia Mecânica - \\ POLI \& COPPE, Universidade Federal do Rio de Janeiro/UFRJ, Centro de Tecnologia, \\ Cidade Universitária, Caixa Postal 68503, 21945-970 Rio de Janeiro, RJ, Brasil \\ ${ }^{3}$ Politehnica University of Bucharest, Applied Thermodynamic Chair, 313 Splaiul, \\ Independentei, 77206 Bucharest, Romania
}

Manuscript received on August 22, 2014; accepted for publication on February 13, 2015

\begin{abstract}
This paper aims to renew interest on mixed thermal convection research and to emphasize three issues that arise from the present analysis: (i) a clear definition of the reference temperature in the Boussinesq approximation; (ii) a practical delimitation of the three convective modes, which are the forced convection (FC), mixed convection (MC) and natural (or free) convection (NC); (iii) and, finally, a uniform description of the set $\mathrm{FC} / \mathrm{MC} / \mathrm{NC}$ in the similarity framework. The planar case, for which analytical solutions are available, allows a detailed illustration of the answers here advanced to the above issues.
\end{abstract}

Key words: mixed convection, Boussinesq approximation, similarity, buoyancy forces, entropy production, reverse flow.

\section{INTRODUCTION}

\section{FUNDAMENTALS OF MiXED CONVECTION}

In most of the works on convective heat transfer, (for instance Bejan 2004, Gebhart et al. 1988, Incropera and De Witt 1985, Kakaç and Yener 1995, Kaviany 1994, Padet 2010, Taine and Petit 1989), the mixed convection is shortly treated relative to the forced, natural, phase change convection modes, or sometimes simply omitted. This reality is related to the fact that mixed convection is a complex subject for which many elements are missing for a general and coherent approach.

Correspondence to: Jacques Padet

E-mail: jacques.padet@univ-reims.fr
This heat or mass transfer mechanism relies in fact on two types of mechanical forces: the pressure forces (generated by a pressure gradient $\overrightarrow{\operatorname{grad}} p^{*}$ ) and the buoyancy forces (generated by a variable density in the gravitational field). The latter are due either to the fluid dilatability as a function of temperature (thermal convection), or to a variation of species concentration in a mixture (mass convection).

In practical problems, the simultaneous consideration of the two forces is frequently avoided and two simpler convection modes are defined:

- natural or free convection (NC), when buoyancy forces are dominant 
- forced convection (FC), when buoyancy forces are negligible.

Natural and forced convection are, thus, limiting cases for the more general convection model, usually called mixed convection (MC).

For a dilatable, non-isothermal fluid flow, the governing equations expressing the steady-state conservation of mass, momentum, and energy are as follows, respectively:

$$
\begin{aligned}
& \operatorname{div} \rho \vec{V}=0 \\
& \overline{\overline{\operatorname{grad}}} \vec{V} . \rho \vec{V}=\rho \vec{g}-\overrightarrow{g r a d} p \\
& \quad+\mu\left(\Delta \vec{V}+\frac{1}{3} \overrightarrow{\operatorname{grad}} \operatorname{div} \vec{V}\right) \\
& \quad \rho C_{p}(\vec{V} \cdot \overrightarrow{g r a d} T)=\Phi+\lambda \Delta T
\end{aligned}
$$

where $\overline{\overline{g r a d}} \vec{V}$ is the tensor gradient of the velocity field (components $\partial V_{i} / \partial x_{j}$ ), and where the symbol $\Delta$ represents here the Laplacian operator (sum of second space derivatives).

In general, the problem is simplified by neglecting the dissipation function $\Phi$ and by adopting the so called Boussinesq approximation, which basically includes three steps:

- $\mathrm{B}_{1}$ : The density variations are assumed negligible, except in the gravity force term

- $\mathrm{B}_{2}$ : In this gravity term, the effect of pressure on the density is neglected, compared to that of temperature

- $\mathrm{B}_{3}$ : Density $\rho(T)$ is expressed as a linear function of the temperature in the vicinity of a reference value $T_{\text {ref }}$, as:

$$
\rho=\rho_{\text {ref }}\left[1-\beta\left(T-T_{r e f}\right)\right]
$$

where $\rho_{\text {ref }}$ and $\beta$ are the density and the thermal expansion coefficient at $T_{\text {ref }}$.

By introducing the driving pressure $p *$ defined as:

$$
\overrightarrow{\operatorname{grad}} \rho^{*}=\overrightarrow{\operatorname{grad}} p-\rho \vec{g}
$$

equation (2) becomes, after division by $\rho$ (the difference $\rho-\rho_{\text {ref }}$ being negligible according to B1): $\overline{\overline{\operatorname{grad}}} \vec{V} \cdot \vec{V}=$

$-\beta\left(T-T_{r e f}\right) \vec{g}-\frac{1}{\rho} \overrightarrow{g r a d} p^{*}+v \Delta \vec{V}$
(a)
(b)
(c)

(notation of the source terms by (a), (b), (c) will be used later).

Equation (3) is thus reduced to:

$$
\vec{V} \cdot \overrightarrow{g r a d} T=a \Delta T
$$

THREE FUNDAMENTAL ISSUES

From the previous presentation, three questions are raised, for which some answers are suggested in this paper.

\section{Practical characterization of $F C$ and $N C$}

At first, forced and natural convection have been defined as theoretical limiting cases of the actual convective transfer phenomenon, but the practical "frontiers" beyond which the limiting approaches may be used, must inevitably be adopted as arbitrary and conventional limits. The pure NC (zero pressure gradient) and the pure FC (zero buoyancy forces) are ideal cases, while dominant $\mathrm{NC}$ and dominant FC (simply called $\mathrm{NC}$ and FC) must be characterized by clear criteria based on the associated physics. Unfortunately, the existing literature provides fragmentary indications and these are poorly sustained by means of distinguishing among the three possible convective regimes.

\section{Boussinesq approximation and the reference temperature}

In second place, it can be noticed that the three terms (a), (b) and (c) in equation (6) are easy to compare due to the Boussinesq approximation, and especially after adopting the linearized form of $\rho(T)$, but a clear definition of the reference temperature $T_{\text {ref }}$ is then needed. Different authors previously treated this question (Barletta and Zanchini 1999, Wang et al. 2003, Padet 2010, Timshenko 2010) but it seems that no answer of general applicability has yet been given. 
Similarity: compatibility of descriptions in $M C$ and $N C$

The third question concerns the nondimensional description of the three convection modes, using similarity criteria (or dimensionless numbers). The reference flow velocity may be the bulk velocity in internal flows or the freestream velocity in boundary layer flows. But these values might be zero or close to zero in natural convection currents and then a reference velocity is defined based on the thermal buoyancy. The consequence is that the description of the MC, which is based on the Richardson, Reynolds and Peclet numbers, has nothing to do with the description of the NC, which is based on the Grashof and Boussinesq numbers (Padet 2011).

In addition, another difficulty is related to the bulk temperature generally used to describe the heat transfer rates. It would be of interest then to reconsider the reference values and examine if a true continuity may be introduced for the transition MC-NC.

\section{ANALYSIS}

\section{MiXED CONVECTION «FRONTIERS»}

It is obvious that the governing equations (6) and (7) do not indicate any physical limit between the three convection modes. Then, the practical need to distinguish between FC, MC and NC is purely numerical and a question arises: what criterion should be used to adopt an approach like the following?

- the convection is forced if the buoyancy term (a) is less than $x \%$ of the viscous forces term (c)

- the convection is natural if the buoyancy term (a) is more than $y \%$ of the viscous forces term (c)

The values of $x$ and $y$ are obviously arbitrary and must be choosen such as to be accepted in practice. On the other hand, other comparison elements may be prefered, such as pressure drop, kinetic energy, wall friction, dissipation, or entropy production, as it will be shown later in this study.
In order to answer to these questions, the terms in the momentum conservation equation (6) must be compared using, not the local values, but the space-averaged ones, over the flow cross section. More precisely, an arithmetic average would not seem representative, as the local values can be positive or negative. A better comparison may be based on the quadratic averages $\overline{a^{2}}, \overline{b^{2}}$ and $\overline{c^{2}}$.

REFERENCE TEMPERATURE $T_{\text {ref }}$ IN THE BOUSSINESQ

APPROXIMATION

However, before we proceed, a clear definition of the reference temperature is essential, regardless of the approach undertaken for the purpose of this study, as the choice of $T_{\text {ref }}$ can be of great influence on the computed values. It will be chosen here to base the definition on the density, rather than on the temperature itself.

The vectorial equation (6), when projected on the horizontal axis, looses the buoyancy term, while when projected on the upward vertical axis, this term becomes $\beta\left(T-T_{\text {ref }}\right) g$.

The fluid mass in a volume $V$ in the flow may be calculated as:

$$
\begin{aligned}
m & =\int_{V} \rho d V \\
& =\int_{V}\left(\rho-\rho_{r e f}\right) d V+\int_{V} \rho_{r e f} d V
\end{aligned}
$$

or, using the Boussinesq approximation:

$$
m=-\int_{V} \rho_{r e f} \beta\left(T-T_{r e f}\right) d V+\rho_{r e f} V
$$

where the last term is the mass of the same fluid at the reference temperature:

$$
m_{r e f}=\rho_{r e f} V
$$

The resulting buoyancy force applied to volume $V$ is then the difference in weight between the considered fluid and the fluid at $T_{\text {ref }}$.

$$
\begin{aligned}
& \left(m-m_{r e f}\right) \vec{g}=-\vec{g} \int_{V} \rho_{r e f} \beta\left(T-T_{r e f}\right) d V= \\
& -\rho_{r e f} V \beta \vec{g}\left(\frac{1}{V} \int_{V} T d V-T_{r e f}\right)
\end{aligned}
$$


where $\frac{1}{V} \int_{V} T d V=\bar{T}$ is the mean temperature in volume $V$.

These considerations are now applied to the two particular representative groups of systems.

\section{Open systems}

A simple open system is the flue gas channel of a fireplace, whose walls are at imposed constant temperatures. The ambient air is at temperature $T_{a}$. The buoyancy force is generated by the difference in weight between the ambient cold air and the inside hot gas, regardless of the type of flow (Archimedes' law). Thus, the reference values for the Boussinesq approximation are:

$$
\rho_{\text {ref }}=\rho_{a} ; T_{r e f}=T_{a}
$$

Nothing is modified if the channel is bounded by two walls whose temperatures are different, and if the distance between the walls is increased so that to achieve a boundary layer flow at each of them. In any of these cases, the reference temperature remains the ambient temperature $T_{a}$.

\section{Closed or infinite systems}

In closed systems (like a differentially heated enclosure) or infinite systems (like a fully developed flow with no entrance or exit conditions, cf. below), the concept of ambient has no meaning and another reference value must be defined based on the entire system. Two commonly encoutered systems are considered here.

(i) For a vertical rectangular enclosure (of volume $V=A H$, with depth $W=1$ ), whose walls are at uniform temperatures $T_{1}>T_{2}$, the convection is purely natural and the balance of the buoyancy forces is zero over $V$ (eq. 8c), so that:

$$
T_{r e f}=\frac{1}{V} \int_{V} T d V-\bar{T} \cong \frac{T_{1}+T_{2}}{2}
$$

This value is in agreement with Timshenko's conclusion, obtained in a different way (Timshenko 2010).
If the vertical axis is " $x$ ", the above equation can be expressed with a mean temperature over the horizontal cross sectional area:

$$
T_{\text {ref }}=\frac{1}{H} \frac{1}{A} \int_{H} \int_{A} T d A d x
$$

(ii) The fully developed flow between two walls of uniform temperatures $T_{1}>T_{2}$ is another common convection problem.

- in natural convection, this configuration corresponds to an enclosure of infinite height, with a temperature independent of $x$. Then:

$$
T_{\text {ref }}=\frac{1}{A} \int_{A} T d A
$$

i.e. $T_{\text {ref }}$ is the space-averaged temperature over the horizontal cross-section area of the flow.

The reference density $\rho_{\text {ref }}$ results as the mean value in a slice of unitary height, such as:

$$
m=m_{\text {ref }}=\rho_{\text {ref }} A \times 1=\rho_{\text {ref }} A
$$

- in mixed convection, nothing has to be changed in the previous reasoning, and definition (9c) is conserved.

The reference temperature, as defined by eqs. (9a,b,c), was sometimes empirically defined in the literature, and even estimated based on mathematical (Barletta and Zanchini 1999) or symmetry considerations (Padet 2010, ch.6). Here, its expression was derived based simply on physics and is equally valid for steady or dynamic regimes.

\section{SIMILARITY AND CONVECTION}

\section{Similarity criteria in mixed convection}

Since long time ago, the non-dimensionalization of governing equations became normal, inducing sometimes confusion between dimensionless numbers and similarity criteria. A similarity criterion is a number without dimension, but a dimensionless number is not necessarily a similarity criterion. For example, the Nusselt and Rayleigh numbers are not similarity criteria, although they are frequently treated as such (Padet 2011, ch.2). 
By considering only the momentum conservation equation in convection problems, a rigorous similarity approach brings up the dimensionless expressions named as Richardson (Ri), Reynolds $(R e)$, Euler $(E u)$, and Grashof $(G r)$ numbers (in equations (10) and (11) below, the symbol ${ }^{+}$denotes dimensionless values, the LHS terms representing momentum fluxes and the RHS terms being momentum sources, i.e. forces applied to the fluid).

- In mixed convection, the dimensionless equations and similarity criteria are:

$$
\begin{aligned}
& \overline{\overline{\operatorname{grad}}} \overrightarrow{V^{+}} \cdot \overrightarrow{V^{+}}=R i T^{+} \overrightarrow{g^{+}} \\
& -\operatorname{Eu} \overrightarrow{\operatorname{grad}}\left(p^{*}\right)^{+}+\frac{1}{\operatorname{Re}} \Delta \overrightarrow{V^{+}}
\end{aligned}
$$

* $\boldsymbol{R} \boldsymbol{i}$ : relative to the buoyancy forces

* Eu: relative to the pressure gradient

* $1 / \boldsymbol{R} e$ : relative to viscous forces

defined as:

$R i=\frac{g \beta \Delta T^{0} L^{0}}{\left(V^{0}\right)^{2}} ; E u=\frac{p^{0}}{\rho\left(V^{0}\right)^{2}} ; R e=\frac{V^{0} L^{0}}{v}$

where superscript ${ }^{0}$ means "reference value"; for instance, in internal flows, $L^{0}$ is the hydraulic diameter, and $V^{0}$ is the bulk velocity.

- In natural convection, the dimensionless equation and similarity criteria are:

$$
\begin{aligned}
& \overline{\overline{\operatorname{grad}}} \overrightarrow{V^{+}} \cdot \overrightarrow{V^{+}}=T^{+} \overrightarrow{g^{+}} \\
& +E u_{n} \overrightarrow{\operatorname{grad}}\left(p^{*}\right)^{+}+\frac{1}{G r^{1 / 2}} \Delta \overrightarrow{V^{+}}
\end{aligned}
$$

$* 1$ : relative to the buoyancy forces

${ }^{*} E u_{n}$ : relative to the pressure forces (id 10b)

$* 1 / G r^{1 / 2}$ : relative to the viscous forces;

$$
G r=\frac{\left(V^{0}\right)^{2}\left(L^{0}\right)^{2}}{v^{2}}
$$

where $V^{0}=\left(g \beta \Delta T^{0} L^{0}\right)^{1 / 2}$ in $E u_{n}$ and $G r$.

- In pure natural convection (defined as the special case where $\overrightarrow{\operatorname{grad}} p^{*}=0$ ), eq. (11a) reduces to:

$$
\overline{\overline{g r a d}} \overrightarrow{V^{+}} \cdot \overrightarrow{V^{+}}=T^{+} \overrightarrow{g^{+}}+\frac{1}{G r^{1 / 2}} \Delta \overrightarrow{V^{+}}
$$

It can be noticed here that, as regard to similarity, a natural convection flow inside a channel could be considered as a case of mixed convection, since the bulk velocity inside this channel can be used as reference velocity $V^{0}$.

\section{Comparison of sources}

The first question suggests a comparison of the momentum sources, namely the existing forces in the fluid flow. A similarity criterion $\Gamma$ is defined as (Padet 2011, ch. 2):

$$
\Gamma=\frac{\text { reference flux at the source }}{\text { reference flux transported by the fluid }}
$$

Then, for two sources (forces) denoted as 1 and 2, the denominator being the same:

$$
\frac{\Gamma_{1}}{\Gamma_{2}}=\frac{\text { reference flux at source } 1}{\text { reference flux at source } 2}
$$

With regards to eq. (10), the order of magnitude of the ratio between the buoyancy forces and the viscosity forces is given by:

$$
\frac{R i}{1 / R e}=R i R e
$$

where the product $R i R e$ is named the buoyancy ratio (or coefficient). It is then expected to have this coefficient in the ratio $\overline{a^{2}} / \overline{c^{2}}$ of the terms in eq. (6).

In the same manner, the ratio between the buoyancy forces and the pressure forces $\overline{a^{2}} / \overline{b^{2}}$ will be expressed as a function of the product:

$$
\mathrm{Ri} / \mathrm{Eu}
$$

Then, contrary to what is usually stated in the literature, the Richardson number alone is not representative for the Archimedes forces significance in the momentum balance, this role belonging to the combinations Ri Re or Ri/Eu. This confusion comes from the classical choice for the reference pressure in the dimensionless values, namely, a dynamic pressure: in this case, the Euler number is equal 
to unity. Acceptable in forced convection, such a choice becomes illicit in mixed convection (as it will be shown for a particular case below).

Moreover, a second reason for such a confusion lies in the fact that another dimensionless number, also called Richardson number, is used for characterizing the stability of a stratified fluid, especially in micrometeorology (Padet 2013, Rubin and Atkinson 2001, Sutton 1953).

A similar reasoning applied to the energy conservation equation shows that the order of magnitude of the ratio between the viscous dissipation and the heat flux is given by the Brinkman number $(\mathrm{Br})$, which is the product between the Eckert number $(E c)$ and the Prandtl number $(P r)$, as shown later in this paper.

Non-continuity of the description from MC to NC

For the third question, it is necessary to reconsider the definition of certain reference values used in the similarity criteria, in parallel with the questioning whether the Grashof number should be maintained; this number materializes the discontinuity between MC and NC, which can be observed in eqs. (10) and (11).

\section{METHODS}

\section{FUNDAMENTALS}

In order to clarify the questions raised above, it is useful to consider first sufficiently simple configurations that allow for analytical solutions. Thus, a fully developed two-dimensional flow in the $x$-direction is chosen, for which:

$$
\left.\begin{array}{l}
\partial U / \partial x=0 \\
\partial T / \partial x=0 \\
\partial p^{*} / \partial x=c t e ; \partial p^{*} / \partial y=0
\end{array}\right\}
$$

These assumptions are valid for fluid flows between infinite walls 1 and 2, having uniform temperatures $T_{1}$ and $T_{2}$. This type of system is often treated theoretically (Aung and Worku 1986, Barletta 1998, Barletta and Zanchini 1999, Boulama and Galanis 2004, Guyon et al. 1991, Bayazitoglu et al. 2007).
Definition of $\mathrm{T}_{\text {ref }}$ : A CONSEQUENCE

It is noticed from eqs. (8) and (9) that the balance of the buoyancy forces is zero over the fluid domain:

$$
\int_{A} \rho_{r e f} \beta\left(T-T_{r e f}\right) d A=0
$$

By using eqs. (13), the integration of eq. (6) over $A$ becomes:

$$
0=-\frac{1}{\rho} \int_{A} \frac{d p^{*}}{d x} d A+v \int_{A} \Delta U d A
$$

If $L$ is the perimeter of $A, d l$ the elementary length of $L$, and $\tau_{s}$ the surface viscous shear stress, the balance ot the forces applied to the fluid is the following:

$$
A \frac{d p^{*}}{d x}=-\int_{L} \tau_{s} d l
$$

When the flow is confined between walls of different lengths, $L_{1}$ and $L_{2}$, each with uniform shear stress, it can be written:

$$
L_{1} \tau_{s 1}+L_{2} \tau_{s 2}=-A \frac{d p^{*}}{d x}
$$

It means that, the balance of the buoyancy forces being zero over the fluid domain, the pressure forces are balanced by the surface viscous forces, like in FC. At last, in pure NC, eq.(15c) is reduced to:

$$
L_{1} \tau_{s 1}+L_{2} \tau_{s 2}=0
$$

A consequence of this property is the coexistence of an upward flow and a downward flow, due to the fact that the wall stresses are of opposite signs (Jeng et al. 1992).

\section{EXAMPLE: planar geometry, steady regime}

The system chosen for exemplification (Aung and Worku 1986, Barletta and Zanchini 1999, Guyon et al. 1991, Padet 2010), is a laminar non-isothermal flow between two parallel vertical plates, placed 
at $y=0$ and $y=e$ and having imposed uniform temperatures $T_{1}$ and $T_{2}$, respectively (fig. 1). The fluid is isochor and the flow is globally ascendant (along $x$-coordinate) with a mean (bulk) velocity $V_{m}$. In addition, the driving pressure gradient $d p * / d x$ is constant (eq. 13).

\section{TEMPERATURE AND VELOCITY FIELDS}

The plates are assumed infinite, and the temperature and velocity fields are $x$-independent. So, the immediate result is that the $y$-velocity component, $V$, is zero everywhere. The conservation equations (6) and (7) become:

$$
\begin{gathered}
0=g \beta\left(T-T_{r e f}\right)-\frac{1}{\rho} \frac{d p^{*}}{d x}+v \frac{d^{2} U}{d y^{2}} \\
\frac{d^{2} T}{d y^{2}}=0
\end{gathered}
$$

with boundary conditions:

$$
\left.\begin{array}{lll}
y=0: & T=T_{1} ; & U=0 \\
y=e: & T=T_{2} ; & U=0
\end{array}\right\}
$$

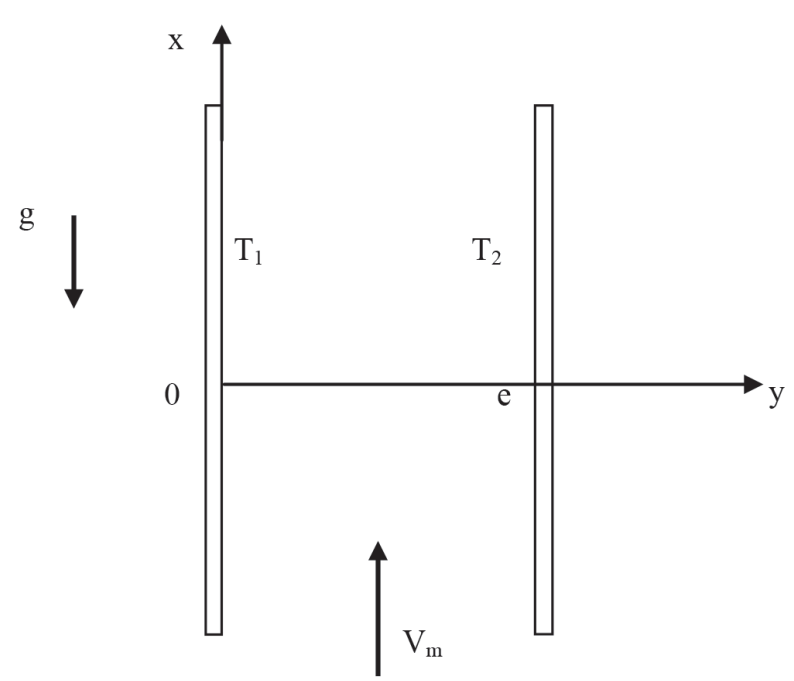

Figure 1 - Plane fully developed flow; $T_{1}-T_{2}=\Delta T>0$.

Thus, the resulting temperature distribution is linear. By denoting $T_{1}-T_{2}=\Delta T$, the solution is as follows:

$$
T=-\frac{\Delta T}{e} y+T_{1}
$$

According to eq. (9), here the reference temperature related to the Boussinesq approximation is:

$$
T_{r e f}=\frac{T_{1}+T_{2}}{2}
$$

Under these conditions, the velocity field derived from eq. (16a) is given by a third order polynomial:

$$
\begin{aligned}
U= & \frac{g \beta}{6 v e} \Delta T y^{3}+\left(-\frac{g \beta}{4 v} \Delta T+\frac{1}{2 \mu} \frac{d p^{*}}{d x}\right) y^{2} \\
& +\left(\frac{g \beta}{12 v} \Delta T-\frac{1}{2 \mu} \frac{d p^{*}}{d x}\right) e y
\end{aligned}
$$

The Boussinesq approximation allows the separation in eq. (19) of the terms $d p * / d x$ with (characteristic to the pure FC) and the terms including $g \beta$ (characteristic to the pure NC). Thus, the velocity can be written as the sum of a FC component and a $\mathrm{NC}$ component:

$$
U=U_{f}+U_{n}
$$

where:

$$
\begin{gathered}
U_{f}=\frac{1}{2 \mu} \frac{d p^{*}}{d x}\left(y^{2}-e y\right) \\
U_{n}=\frac{g \beta \Delta T}{v}\left(\frac{y^{3}}{6 e}-\frac{y^{2}}{4}+\frac{e y}{12}\right)
\end{gathered}
$$

The bulk velocity is computed based on eq. (19) as:

$$
V_{m}=\frac{1}{e} \int_{0}^{e} U d y=-\frac{e^{2}}{12 \mu} \frac{d p^{*}}{d x}
$$

and the volumetric flow rate is given by:

$$
q_{v}=V_{m} e=-\frac{e^{3}}{12 \mu} \frac{d p^{*}}{d x}
$$

The velocity field can also be expressed as a function of the bulk velocity $V_{m}$ : 


$$
\begin{aligned}
U= & \frac{g \beta}{6 v e} \Delta T y^{3}-\left(\frac{g \beta}{4 v} \Delta T+\frac{6 V_{m}}{e^{2}}\right) y^{2} \\
& +\left(\frac{g \beta}{12 v} \Delta T+\frac{6 V_{m}}{e^{2}}\right) e y
\end{aligned}
$$

The velocity gradients at the wall surfaces can now be easily calculated:

- at the hot surface $(y=0)$ :

$$
\left(\frac{d U}{d y}\right)_{y=0}=\frac{g \beta}{12 v} \Delta T e+6 \frac{V_{m}}{e}
$$

- at the cold surface $(y=e)$ :

$$
\left(\frac{d U}{d y}\right)_{y=e}=\frac{g \beta}{12 v} \Delta T e-6 \frac{V_{m}}{e}
$$

\section{RESULTS}

A first remark is that the thermal buoyancy does not affect the bulk velocity and the flow rate. This means that the complementary flow initiated at the hot surface by the buoyancy forces is exactly compensated by the flow deficit caused at the cold surface.

The observation of eq. (20b) allows for the following additional remarks:

- in pure FC, without buoyancy forces, the velocity profile $U_{f}$ is the classical second order polynomial

- in pure NC, the velocity profile $U_{n}$ is antisymmetric and crosses zero at the channel centerline (fig. 2), showing a reverse flow at the cold surface, so that the bulk velocity is zero.

The critical case that indicates the transition to a reverse flow may be inferred from eq. (23b) by imposing a zero velocity gradient at the cold surface. The critical bulk velocity yields, thus, the expression:

$$
V_{m c}=\frac{g \beta \Delta T e^{2}}{72 v}
$$

It is also interesting to consider the mechanical frame of the flow. Then, the friction at the cold wall (23b) can be written as:

$$
\tau_{s e}=\mu\left(\frac{d U}{d y}\right)_{y=e}=\frac{\rho g \beta \Delta T}{12} e-6 \frac{\mu V_{m}}{e}
$$

As for the velocity field, it can be observed that the total friction force is the sum of a force $\tau_{s n}$ due to the gravity field (NC) and of a force $\tau_{s f}$ related to the bulk velocity (FC):

$$
\tau_{s e}=\tau_{s n}-\tau_{s f}
$$

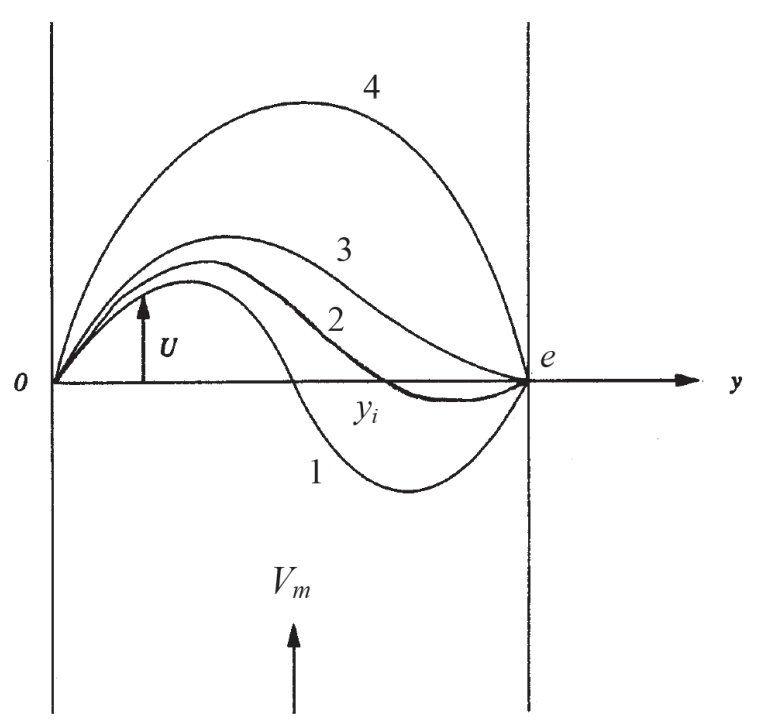

Figure 2 - Velocity fields - 1. Pure NC; 2. Reverse flow $($ RiRe $>288) ; 3$. RiRe $=(\operatorname{RiRe})_{c}=288 ; 4$. Pure FC.

Then a reverse flow appears when the buoyancy friction becomes dominant, the transition corresponding to $\tau_{s e}=0$.

Similarly, it can be written that the friction factor $C_{f} / 2$ includes a buoyancy component and a forced convection component, as:

$$
\frac{1}{2} C_{f e}=\frac{\tau_{s e}}{\rho V_{m}^{2}}=\frac{1}{2} C_{f n}-\frac{1}{2} C_{f f}
$$

In the present case, the hydraulic diameter $D_{h}=2 e$. According to eq. (24b), eq. (24a) becomes:

$$
\frac{1}{2} C_{f e}=\frac{1}{24} \frac{g \beta \Delta T D_{h}}{V_{m}^{2}}-12 \frac{\mu}{\rho V_{m} D_{h}}
$$

This expression contains the Richardson and Reynolds numbers (eq. 10b, with, $V^{0}=V_{m}, L^{0}=D_{h}$ ), and can be written as: 


$$
\frac{1}{2} C_{f e}=\frac{R i}{24}-\frac{12}{R e}
$$

The value $C_{f e}=0$ characterizes a limit between two kinds of flows; the buoyancy ratio has then a critical value $(R i R e)_{c}$ given by:

$$
\frac{R i}{24}-\frac{12}{R e}=0 \Rightarrow(R i R e)_{c}=288
$$

where:

$$
R i \operatorname{Re}=\frac{g \beta \Delta T D_{h}^{2}}{V_{m} v}=\frac{g \beta \Delta T}{V_{m} v} 4 e^{2}
$$

It results that the existence of a reverse flow is governed solely by the thermal buoyancy ratio, and this coefficient has a critical value for the system considered here: $(\text { RiRe })_{c}=288$.

With different boundary conditions, (Desrayaud and Lauriat 2009) and (Kholai et al. 2010), have obtained numerical results indicating a single critical value a little bit less than 300 . This result could then not be restricted only to the case considered in the present study.

A flash-back to the reference temperature seems now useful. As regard to the flow between two parallel walls, some authors choose for $T_{\text {ref }}$ the value corresponding to the cold wall: $T_{\text {ref }}=T_{2}$. But, then, the equilibrium condition expressed by eq. (15c) is no longer respected. In particular, for pure $\mathrm{NC}$ (eq. 15d), the calculation leads to a non-zero volumetric rate (the upward flow rate is superior to the downward flow rate), the flow velocity symmetry being destroyed. It was exactly this symmetry condition, which is physically expected, that was used by Padet (Padet 2010) in reasoning the reference temperature defined by eq. (18). Therefore, the Boussinesq approximation must be used with precaution, especially since the use of nondimensional values aids in hiding this problem.

\section{ENERGY DISSIPATION AND ENTROPY PRODUCTION}

For a long period of time, the energy system optimization was based on energy criteria. However, more recently, the interest moved towards exergy or entropy analysis. In particular, the type of analysis based on entropy production was born in association with irreversible thermodynamics (Glansdorf and Prigogine 1971). But this theory, conceptually very rich, did not offer to the engineering sciences the expected benefits, especially because of its complexity and lack of practical impact. Fortunately, it was re-launched by the works of (Bejan 1994, 1996) who used a simpler and more pragmatical approach for the optimization based on entropy production limitation. Most often, previous works are related to forced convection problems (Herpe 2007, Herpe et al. 2007, Mladin et al. 2006, Stanciu et al. 2005), and rarely to mixed convection problems (Ben Mansour et al. 2005, Zanchini 1998). The extension to mixed convection will be combined in this study with the research of usable criteria to distinguish between $\mathrm{FC}, \mathrm{MC}$ and $\mathrm{NC}$ heat transfer.

It is assumed in what follows that the viscous dissipation influence on the temperature field is negligible, fact justified in the considered examples by Brinkman numbers [eq. (55c)] of order $10^{-7}$. As a consequence, the temperature distribution will remain linear [eq. (17)], decoupled from the velocity field.

\section{Viscous dissipation}

The volumetric (local) dissipation source $\Phi^{\prime \prime \prime}$ is defined by:

$$
\Phi^{\prime \prime \prime}(y)=\mu\left(\frac{d U}{d y}\right)^{2} \quad\left[W / m^{3}\right]
$$

The surface dissipation $\Phi^{\prime \prime}$ results from integration over the flow width:

$$
\Phi^{\prime \prime}=\mu \int_{0}^{e}\left(\frac{d U}{d y}\right)^{2} d y \quad\left[W / m^{2}\right]
$$

By using eq. (22) for the velocity distribution, the above expression becomes: 


$$
\begin{aligned}
\Phi^{\prime \prime}= & 1.39 \times 10^{-3} \frac{(\rho g \beta \Delta T)^{2} e^{3}}{\mu} \\
& +12 \mu \frac{V_{m}^{2}}{e} \quad\left[\mathrm{~W} / \mathrm{m}^{2}\right]
\end{aligned}
$$

indicating that dissipation takes place due to the buoyancy effects (term with $\Delta T$ ), in addition to the dissipation that occurs in the isothermal flow.

The surface dissipation $\Phi^{\prime \prime}$ can also be expressed in dimensionless form by adopting as a reference the surface dissipation for isothermal flow, $\Phi^{\prime \prime}$ :

$$
\Phi^{\prime \prime \circ}=12 \mu \frac{V_{m}^{2}}{e} \quad\left[W / m^{2}\right]
$$

Then, the dimensionless viscous dissipation $\Phi^{+}$is:

$\Phi^{+}=\frac{\Phi^{\prime \prime}}{\Phi^{\prime \prime 0}}=\frac{1.39 \times 10^{-3}}{12} \frac{(\rho g \beta \Delta T)^{2} e^{4}}{\mu^{2} V_{m}^{2}}+1$

It is interesting to note that eq. (29a) includes the thermal buoyancy ratio (25e), and $\Phi^{+}$may also be written as:

$$
\Phi^{+}=7.23 \times 10^{-6}(\text { Ri Re })^{2}+1
$$

\section{Entropy production}

When considering the entropy production in mixed convection, a distinction must be made between the viscous source $\Phi^{\prime \prime}(27 \mathrm{a})$ that produces "viscous entropy" $\sigma_{v}^{\prime \prime}\left[W / m^{2} K\right]$, and the temperature gradient between the walls, that originates "thermal entropy", $\sigma_{t h}^{\prime \prime}\left[W / m^{2} K\right]$.

The viscous entropy produced in a horizontal section of the flow (fig. 1) is calculated using the local dissipation source of eq. (26):

$$
\sigma_{v}^{\prime \prime}=\int_{0}^{e} \frac{\Phi^{\prime \prime \prime}(y)}{T(y)} d y \quad\left[W / m^{2} K\right]
$$

where $T$ is the absolute temperature.

In first approximation, for mild temperature differences $(\Delta T / T<10 \%)$, the production of viscous entropy may be expressed by reporting the total dissipation of eq. (27b) to the reference temperature $T_{\text {ref }}$ :

$$
\begin{aligned}
\sigma_{v}^{\prime \prime}= & \frac{\Phi^{\prime \prime}}{T_{r e f}}=1.39 \times 10^{-3} \frac{(\rho g \beta \Delta T)^{2} e^{3}}{\mu T_{r e f}} \\
& +12 \frac{\mu V_{m}^{2}}{e T_{r e f}}
\end{aligned}
$$

Once more, the Boussinesq approximation allows for distinguishing the terms related to either the buoyancy or to the fluid viscosity.

The local thermal entropy production $\sigma_{t h}^{\prime \prime \prime}$ $\left[\mathrm{W} / \mathrm{m}^{3} \mathrm{~K}\right]$ is given by:

$$
\sigma_{t h}^{\prime \prime \prime}(y) \frac{\lambda}{T^{2}}\left(\frac{d T}{d y}\right)^{2}
$$

For the example considered here, the temperature gradient is constant and equal to $(\Delta T / e)$, leading to the total thermal entropy production in a horizontal section of the flow $\sigma_{\text {th }}^{\prime \prime}\left[\mathrm{W} / \mathrm{m}^{2} \mathrm{~K}\right]$ :

$$
\sigma_{t h}^{\prime \prime}=\int_{0}^{e} \sigma_{t h}^{\prime \prime \prime}(y) d y=\lambda\left(\frac{\Delta T}{e}\right)^{2} \int_{0}^{e} \frac{1}{T^{2}} d y
$$

This expression is also evaluated in a first approximation, by assuming that:

$$
\sigma_{\text {th, approx }}^{\prime \prime}=\frac{\lambda}{e}\left(\frac{\Delta T}{T_{\text {ref }}}\right)^{2}
$$

The total entropy production $\sigma_{\text {tot }}^{\prime \prime}\left[\mathrm{W} / \mathrm{m}^{2} \mathrm{~K}\right]$ results then as the sum:

$$
\sigma_{\text {tot,approx }}^{\prime \prime}=\sigma_{v}^{\prime \prime}+\sigma_{\text {th,approx }}^{\prime \prime}
$$

or, using eqs. (30) and (32b):

$$
\begin{aligned}
\sigma_{\text {tot, approx }}^{\prime \prime}= & 1.39 \times 10^{-3} \frac{(\rho g \beta \Delta T)^{2} e^{3}}{\mu T_{\text {ref }}} \\
& +\frac{12 \mu V_{m}^{2}}{e T_{\text {ref }}}+\frac{\lambda}{e}\left(\frac{\Delta T}{T_{\text {ref }}}\right)^{2}
\end{aligned}
$$

Figure 3 presents all the terms of eq. (33b) for a laminar water flow at a reference temperature of $20^{\circ} \mathrm{C}$. It is shown that the total entropy is dominated by buoyancy at large values of separation distances $e$, and by heat transfer at small values of $e$. 
Numerical calculations indicated that the approximate value in eq. (33b) of $\sigma_{t o t}^{\prime \prime}$ is very close to its exact value (the differences are inferior to $1 \%$ for $\Delta T$ less than $50 \mathrm{~K}$ ). In addition, it is clear that the contribution of the second term, dependent on the mean velocity $V_{m}$, is negligible for all $e$ values, under laminar flow conditions, as initially assumed.

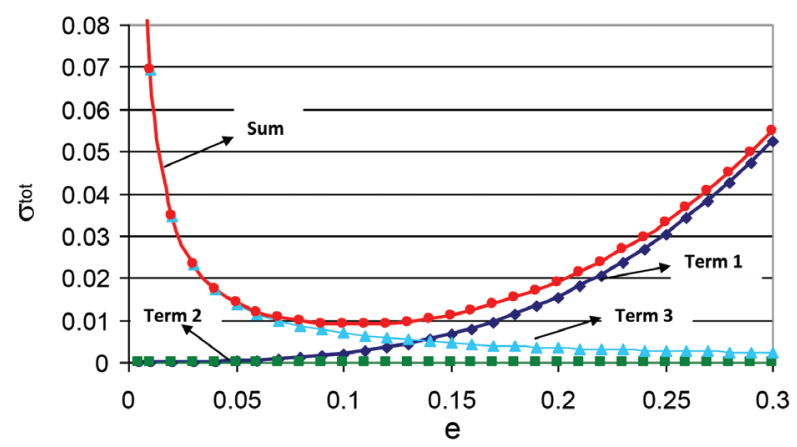

Figure 3 - Buoyancy entropy (1), isothermal entropy (2), thermal entropy (3), total (4). Water flow; $T_{r e f}=20^{\circ} \mathrm{C} ; \Delta T=10^{\circ} \mathrm{C}$; $V_{m}=0.001 \mathrm{~m} / \mathrm{s}$ [eq. $\left.(33 \mathrm{~b})\right]$.

\section{SELECTION CRITERIA FOR CF/CM/CN}

Different criteria to distinguish between forced, mixed and natural convection (Padet 2010, Padet et al. 2004, 2005, 2009) are examined here. They are based either on the acting forces, eq. (16a), on the kinetic energy components (velocity field in eqs. (19) and (20b)), on the dissipation, eq. (27b), or on the entropy production, eq. (33b).

\section{$1^{\text {st }}$ Criterion: comparison of the gravitational and viscous forces}

By using eq.(16a), the terms (a) and (c) in eq. (6) become:

$$
a=g \beta\left(T-T_{r e f}\right) ; c=v \frac{d^{2} U}{d y^{2}}
$$

or, as functions of $y$, with eqs.(17)-(19):

$$
a=g \beta \Delta T\left(\frac{1}{2}-\frac{y}{e}\right)
$$

$$
c=-g \beta \Delta T\left(\frac{1}{2}-\frac{y}{e}\right)-\frac{12 v V_{m}}{e^{2}}
$$

The squared averages over the range $[0, \mathrm{e}]$ are, respectively:

$$
\begin{gathered}
\overline{a^{2}}=\frac{1}{12} g^{2} \beta^{2} \Delta T^{2} \\
\overline{c^{2}}=\frac{1}{12} g^{2} \beta^{2} \Delta T^{2}+144 \frac{v^{2} V_{m}^{2}}{e^{4}}
\end{gathered}
$$

It is noted that, like it was concluded before, their ratio $P^{2}$ is expressed solely as a function of the thermal buoyancy coefficient $R i$ Re defined by eq. (25e):

$$
P^{2}=\frac{\overline{a^{2}}}{\overline{c^{2}}}=\frac{(R i R e)^{2}}{(\operatorname{RiRe})^{2}+27648}
$$

or:

$$
\operatorname{Ri} e=\frac{166.28 P}{\sqrt{1-P^{2}}}
$$

The latter equation indicates that $P$ is a number between 0 and 1 , the limiting situations corresponding to:

$P=0 \quad \Rightarrow \quad$ RiRe $=0 \quad$ : FC

$P \rightarrow 1 \Rightarrow R i R e \rightarrow \infty: \quad \mathrm{NC}$

In order to establish some practical limits, it can be admitted that the convection is mainly natural if the buoyancy forces are less than $5 \%$ relative to the viscous forces (when $P<0.05$ ), and that the convection is mainly forced when they represent at least $95 \%(P>0.95)$. Equation (37b) leads then to the following selection criteria:

$P<0.05 \Rightarrow R i R e<8.3 \quad$ for $F C$

$P>0.95 \Rightarrow R i R e>505.9$ for $N C$

$2^{\text {nd }}$ Criterion: comparison of the gravitational and pressure forces

Referring again to eqs. (6) and (16a), terms ( $a$ ) and (b) of eq. (6) are divided this time using also eq. (35) and noting from eq. (21a) that: 


$$
b=\frac{12 v}{e^{2}} V_{m}=c s t
$$

Then, the ratio $\Gamma^{2}$ of squared mean values is given by:

$$
\Gamma^{2}=\frac{\overline{a^{2}}}{\overline{b^{2}}}=\frac{(R i R e)^{2}}{27648}
$$

Again, this criterion is also a function of RiRe only (as stated in eq. 12d). In fact, in this particular case where the pressure gradient may be expressed as a function of the bulk velocity, eq. (21b), the Euler number becomes ( $p^{* \circ}$ being a reference pressure, equal here to $\left.\left(d p^{*} / d x\right) D_{h}\right)$ :

$$
E u=\frac{p^{* \circ}}{\rho V_{m}^{2}}=\frac{48}{R e}
$$

so that the ratio $\mathrm{Ri} / \mathrm{Eu}$ is proportional to RiRe.

Equation (40a) may be re-written as:

$$
\text { Ri } \operatorname{Re}=166.28 \Gamma
$$

showing that the equilibrium of gravitational and pressure forces $(\Gamma=1)$ is reached when RiRe = 166.28 and not when $R i=1$, as sometimes stated.

But differently to the ratio $P$ in criterion 1, the ratio $\Gamma$ varies from zero to infinity, as $a=0$ in FC and $b=0$ in NC. The distinguishing values are then adapted as follows: it is admitted that, in FC, the buoyancy forces represent maximum $5 \%$ of the pressure forces $(\Gamma<0.05)$, or in $\mathrm{NC}$, the pressure forces are less than $5 \%$ of buoyancy forces $(b<$ $0.05 \sqrt{\overline{a^{2}}}$, or $\Gamma>20$ ). Equation (40a) provides the following bounds:

$$
\begin{array}{llll}
\Gamma<0.05 & \Rightarrow & R i R e<3325.6 & \text { for } N C \\
\Gamma>20 & \Rightarrow & R i R e>8.31 & \text { for } F C
\end{array}
$$

For forced convection, the boundary is about the same as that indicated by the first criterion. However, for natural convection, it is pushed at a much higher value for RiRe. $3^{\text {rd }}$ Criterion: comparison of the friction constraints at the wall surfaces

Another selection criterion may be based on the comparison of the friction constraints at the surfaces, given by eqs. (23a) and (23b). These equations indicate that the velocity gradients are equal in magnitude and of opposite signs in $\mathrm{FC}$, equal in magnitude and of same sign in NC (see fig. 2). Their relative difference may also be used for comparison between the two types of convection. Several trials indicated that a value of $10 \%$ for this difference would represent a good compromise in distinguishing FC and NC. The following mathematical expression is used for the new ratio $K_{f}$ :

$$
\frac{(d U / d y)_{y=0} \pm(d U / d y)_{y=e}}{(d U / d y)_{y=0}}=K_{f}
$$

with a positive sign for $\mathrm{FC}$ and a negative one for $\mathrm{NC}$. By using the velocity profile, the ratio becomes again a function of $R i R e$ only:

- For dominant NC:

$$
K_{f}=\frac{1}{\frac{R i R e}{576}+0.5}
$$

or

$$
\text { Ri } R e=\frac{576}{K_{f}}-288
$$

If admitted $K_{f}<0.1$ :

$$
\text { RiRe }>5472 \text { for } N C
$$

- For dominant FC:

$$
K_{f}=\frac{1}{\frac{144}{R i R e}+0.5}
$$

or

$$
R i \operatorname{Re}=\frac{144}{\frac{1}{K_{f}}-0.5}
$$


If admitted $K_{f}<0.1$ :

$$
\text { RiRe }<15.2 \text { for FC }
$$

Noteworthy, the values of RiRe limiting FC/ $\mathrm{MC}$ and $\mathrm{MC} / \mathrm{NC}$ are of the same order of magnitude as in previous criteria.

$4^{\text {th }}$ Criterion: comparison based on kinetic energy

Another option to distinguish between different types of convection starts from the velocity distribution (20b), where the Boussinesq approximation allows the dissociation of the component $U_{n}$ generated by buoyancy and the component $U_{f}$ generated by the pressure gradient. Similarly, kinetic energy can be devided into $K_{e n}$ and $K_{e f}$ defined as:

$K_{e n}=\frac{\rho}{2 e} \int_{0}^{e} U_{n}^{2} d y, K_{e f}=\frac{\rho}{2 e} \int_{0}^{e} U_{f}^{2} d y$

The comparison of these two terms is in fact the comparison of the mean squared values of $U_{n}$ and $U_{f}$. A new ratio is defined as $K_{e}$ :

$$
K_{e}=\frac{K_{e n}}{K_{e f}+K_{e n}}
$$

which is equal to:

$$
\begin{aligned}
& K_{e}=\frac{R i R e}{\sqrt{580608+(R i R e)^{2}}} \text {, or: } \\
& R i \operatorname{Re}=\frac{762 K_{e}}{\sqrt{1-K_{e}^{2}}}
\end{aligned}
$$

Like coefficient $P, K_{e}$ is a parameter situated in the range $[0,1]$. Even the expressions (39b) and (49) are similar.

By adopting the practical convention that, for natural convection, the gravitational kinetic energy represents at least $95 \%$ of the total kinetic energy $\left(K_{e}>0.95\right)$, while, for forced convection it represents less than $5 \%\left(K_{e}>0.05\right)$, it results in:

$\begin{array}{lll}R i R e>2318 & \text { for } & N C \\ R i R e & <38.15 & \text { for }\end{array}$ $5^{\text {th }}$ Criterion: comparison based on the dissipation The mechanical energy dissipation studied earlier may also be used to select between FC/MC/NC conditions. By looking at the equations (27) and (29), two ways of defining a selection criterion are apparent: compare the dissipation caused by the gravitational field (term with $\Delta T$ or RiRe) with the dissipation in an isothermal flow, or with the total dissipation.

a) First option leads to (see eq. (29)):

$$
\begin{aligned}
K_{d} & =\frac{7.23 \times 10^{-6}(\mathrm{RiRe})^{2}}{1} \\
& =7.23 \times 10^{-6}(\mathrm{RiRe})^{2}
\end{aligned}
$$

This criterion may take values in the range $[0,+\infty]$. Then, it may be agreed that the convection is of forced type if the gravitational dissipation is less than $5 \%$ relative to the isothermal dissipation (i.e. $K_{d}<0.05$ ), or vice-versa, that the convection is natural if the latter is less than $5 \%$ relative to the former (i.e. $K_{d}>1 / 0.05=20$ ). And since equation (49a) may be rewritten as:

$$
R i R e=372 \sqrt{K_{d}}
$$

it is immediately obtained the following:

$K_{d}>20 \Rightarrow \operatorname{RiRe}<1663$
$K_{d}<0.05 \Rightarrow \operatorname{RiR} \operatorname{Ror}>83$

b) Second option leads to the criterion:

$$
K_{d t}=\frac{7.23 \times 10^{-6}(R i R e)^{2}}{7.23 \times 10^{-6}(R i R e)^{2}+1}
$$

which varies in the interval $[0,1]$. It is then reasonable to admit that the convection is forced if the gravitational dissipation is less than $5 \%$ of the total (i.e. $K_{d t}<0.05$ ), and natural when the same term represents more than $95 \%$ relative to the total dissipation (i.e. $K_{d t}>0.95$ ). Equation (51a) may be expressed as:

$$
\operatorname{Ri} \operatorname{Re}=\left[\frac{1}{7.23 \times 10^{-6}} \frac{K_{d t}}{1-K_{d t}}\right]
$$


which results in the following separation domains:

$K_{d t}>0.95 \Rightarrow \operatorname{RiRe}<1621$ for $N C$

$K_{d t}<0.05 \Rightarrow \operatorname{RiRe}>85$ for $F C$

A comparison between the results associated with the first and the second options indicates that they are practically equivalent.

$6^{\text {th }}$ Criterion: comparison based on the entropy production

Another selection criterion occurs naturally as a continuity of the study presented sooner, where the entropy production is quantified.

a) A first possibility is to compare in eq. (33b) buoyancy entropy (first term) and isothermal flow entropy (second term). The resulting ratio is exactly the criterion $K_{d}$ already analyzed with regards to dissipation. Therefore, it is not necessary to define it again in relation to entropy production.

b) A second approach means dividing the buoyancy entropy to the total entropy production:

$K_{S}=\frac{1.39 \times 10^{-3} \frac{(\rho g \beta \Delta T)^{2} e^{3}}{\mu T_{r e f}}}{1.39 \times 10^{-3} \frac{(\rho g \beta \Delta T)^{2} e^{3}}{\mu T_{r e f}}+\frac{12 \mu V_{m}^{2}}{e T_{r e f}}+\frac{\lambda}{e}\left(\frac{\Delta T}{T_{r e f}}\right)^{2}}$

or:

$$
\begin{aligned}
& \frac{1}{K_{S}}=1+8.63 \times 10^{3} \frac{\mu^{2} V_{m}^{2}}{(\rho g \beta \Delta T)^{2} e^{4}}+\frac{1}{T_{r e f}} \\
& \frac{\lambda \mu}{1.39 \times 10^{-3}(\rho g \beta)^{2} e^{4}}
\end{aligned}
$$

This last expression may be equally rewritten in dimensionless form as:

$$
\begin{aligned}
& \frac{1}{K_{S}}=1+\frac{1}{(\operatorname{RiRe})^{2}}\left[1.38 \times 10^{5}+0.115 \times 10^{5}\right. \\
& \left.\frac{\Delta T}{T_{\text {ref }}} \frac{1}{B r}\right]
\end{aligned}
$$

where:

$$
B r=\mu V_{m}^{2} / \lambda \Delta T=\operatorname{Pr} E c
$$

is the Brinkman number.
Approaching this criterion with benchmarks as $K_{S}=0.05$ and $K_{S}=0.95$, like it was done with $K_{d t}$, would lead to expressions that are hard to manipulate, because of the large number of dimensionless parameters, including in particular the ratio $\Delta T / T_{\text {ref }}$ (Bejan 1994). It seems then reasonable to push away the entropy related criteria when it comes to distinguishing among the three convection modes.

\section{Additional remarks}

A general remark over the proposed criteria is that the benchmark values of the product RiRe have the same order of magnitude. Therefore, a priori, each of them seems acceptable.

On the other hand, it is noticed that all the analyzed criteria include the value $\operatorname{RiRe}=288$ indicating the occurrence of a reverse flow on the cold wall, as well as the value $\operatorname{RiRe}=166.28$ which characterizes the equilibrium between pressure and buoyancy forces. It can be seen that the condition for $R i R e=288$ is achieved for $P=$ 0.866 ( $1^{\text {st }}$ criterion), $\Gamma=1.73$ ( $2^{\text {nd }}$ criterion $)$ or for $K_{e}=0.35$ ( $3^{\text {rd }}$ Criterion $)$, while the condition RiRe $=166.28\left(\Gamma=1,2^{\text {nd }}\right.$ criterion $)$ corresponds to $P=$ $0.707\left(1^{\text {st }}\right.$ criterion $)$ or to $K_{e}=0.213\left(3^{\text {rd }}\right.$ criterion $)$.

The definition interval for the criteria $P, \Gamma$, etc may represent the base for a first selection. In fact, it is more comfortable to use values in the range $[0,1]$ than in the range $[0,+\infty]$. This choice leads to the removal of the $2^{\text {nd }}$ and $5^{\text {th }}$ criteria ( $\Gamma$ and $K_{d}$, respectively).

Among the left criteria, $P\left(1^{\text {st }}\right.$ criterion $)$ and $K_{f}$ $\left(3^{\text {rd }}\right.$ criterion) are based on the present forces, while $K_{e}\left(4^{\text {th }}\right.$ criterion $)$ and $K_{d t}\left(5^{\text {th }}\right.$ criterion $)$ are based on mechanical energy. In many applications, the latter are to be preferred. It results then that:

- with $K_{e}: \mathrm{MC} \Leftrightarrow 38<\operatorname{RiRe}<2318$

- with $K_{d t}: \mathrm{MC} \Leftrightarrow 85<\operatorname{RiRe}<1621$

The above values being just benchmarks, and given the fact that simple values are easy to remember and use, it is proposed a compromise between the two criteria and admitted that, in 
practical applications, the mixed convection is characterized by the condition:

$$
M C \Leftrightarrow 50<\operatorname{RiRe}<2000
$$

Otherwise, unless marked precision is required, buoyancy may be neglected if the product RiRe is inferior to 50 (FC is dominant), and pressure gradient is negligible if the product RiRe is superior to 2000 (NC is dominant).

\section{Numerical example}

Consider, for instance, water flow at $30^{\circ} \mathrm{C}(\beta \approx 4$. $\left.10^{-4} \mathrm{~K}^{-1} ; v \approx 8.5 \cdot 10^{-7} \mathrm{~m}^{2} / \mathrm{s}\right)$, with $e=2 \cdot 10^{-2} \mathrm{~m}$, $V_{m}=3 \cdot 10^{-2} \mathrm{~m} / \mathrm{s}, g=9.81 \mathrm{~m} / \mathrm{s}^{2}$. Then:

$$
R e=1412 ; R i=0.174 \Delta T ; R i R e=246 \Delta T
$$

The corresponding temperature differences are, respectively:

RiRe $=50: \Delta T=0.2^{\circ} \mathrm{C}$

RiRe $=2000: \Delta T=8.13^{\circ} \mathrm{C}$

and the presence of a reverse flow $(\operatorname{RiRe}>288)$ corresponds to:

$\Delta T>1.17^{\circ} \mathrm{C}$

\section{CONTINUITY OF THE FC-MC-NC DESCRIPTION}

\section{Critics for two usual concepts and possible} solutions

In the first part, it was underlined the problem raised by the dimensionless description of a flow when it comes close to a pure natural convection mode, as this brings up a discontinuity and imposes the replacement of $R e$ and $R i$ by $G r$. For the case presented here, the difficulty comes from the existence of a reverse flow for RiRe $>288$, or of a recirculation zone in other cases. Then, the mean velocity, which is simply an arithmetic average, is no longer representative for the flow, or at least it only describes the global flow, ignoring the presence of two opposed fluxes. In particular, the mean flow is zero in pure natural convection.
This difficulty has effect on another concept, the bulk temperature, largely used as a reference to describe the convective heat transfer, and expressed by:

$$
T_{m}=\frac{1}{V_{m} e} \int_{0}^{e} T U d y
$$

such as $T_{m} \rightarrow \infty$ when $V_{m} \rightarrow 0$.

In fact, $T_{m}$ is the temperature of an isothermal flow that would transport the same heat rate with the same mean velocity. As a result, in pure $N C$, the transport of a finite energy with a zero velocity needs an infinite temperature. This problem was brought up also by other authors, in particular by (Aung and Worku 1986).

Two approaches allow overcoming these difficulties. One approach, named bi-directional, separates the upward flow from the downward flow and considers, for example, only the upward flow. Another one may be based on the definition of a square mean flow velocity that would replace the mean velocity role in convection description. The two approaches are explored further.

\section{Bi-directional approach}

A way to re-establish the continuity in the dimensionless description of the convection is then to consider only the upward flow. This does not change anything if $R i R e<288$. In the opposite situation, there is a location $y_{i}$ where the flow changes direction (fig. 2), such as:

$$
U\left(y_{i}\right)=0
$$

Equation (22) becomes now a simple second order equation with the roots:

$$
\begin{aligned}
y_{i} & =e \\
y_{i} & =\frac{e}{2}+36 \frac{V_{m} v}{g \beta \Delta T e}
\end{aligned}
$$

or:

$$
y_{i}^{+}=\frac{y_{i}}{e}=\frac{1}{2}+\frac{144}{\operatorname{RiRe}}
$$


For RiRe $>288$, it is evident that $y_{i}<e$. Now, the following can be calculated:

- the upward flow rate:

$$
q_{v \uparrow}=\int_{0}^{y_{i}} U(y) d y
$$

- the mean upward flow velocity:

$$
V_{m \uparrow}=\frac{q_{v}}{y_{i}}
$$

The resulting flow rate is:

$$
\begin{aligned}
& q_{v \uparrow}=\frac{g \beta \Delta T}{24 v e} y_{i}^{4}-\frac{g \beta \Delta T}{12 v} y_{i}^{3} \\
& -\frac{2 V_{m}}{e^{2}} y_{i}^{3}+\frac{g \beta \Delta T e}{24 v} y_{i}^{2} \frac{3 V_{m}}{e} y_{i}^{2}
\end{aligned}
$$

The Reynolds and Richardson numbers corresponding to the upward flow (having the hydraulic equivalent diameter $2 y_{i}$ ) have now non-zero finite values in pure natural convection:

$$
\begin{aligned}
& R e_{\uparrow}=\frac{2 y_{i} V_{m \uparrow}}{v}=\frac{2 q_{v \uparrow}}{v} \\
& R i_{\uparrow}=\frac{g \beta \Delta T 2 y_{i}}{V_{m \uparrow}^{2}}
\end{aligned}
$$

The Reynolds number is given now by:

$$
\begin{aligned}
R e_{\uparrow}= & \frac{g \beta \Delta T}{12 v^{2} e} y_{i}^{4}-\frac{g \beta \Delta T}{6 v^{2}} y_{i}^{3}-\frac{4 V_{m}}{v e^{2}} y_{i}^{3}+ \\
& \frac{g \beta \Delta T e}{12 v^{2}} y_{i}^{2}+\frac{6 V_{m}}{v e} y_{i}^{2}
\end{aligned}
$$

and, with $y_{i}^{+}=y_{i} / e$ :

$$
\begin{gathered}
R e_{\uparrow}=\frac{g \beta \Delta T e^{3}}{v^{2}}\left(\frac{\left(y_{i}^{+}\right)^{4}}{12}-\frac{\left(y_{i}^{+}\right)^{3}}{6}+\frac{\left(y_{i}^{+}\right)^{2}}{12}\right) \\
-\frac{2 V_{m} e}{v}\left(2\left(y_{i}^{+}\right)^{3}-3\left(y_{i}^{+}\right)^{2}\right) \\
\left(G_{2}\right)
\end{gathered}
$$

The two terms $G_{1}$ and $G_{2}$ of eq. (61) are functions of RiRe since $y_{i}^{+}$is a function of this product. In addition, the factors that precede the parenthesis are nothing else, but $\operatorname{RiRe}^{2} / 8$ and Reynolds number $R e$, respectively:
$R e_{\uparrow}=\frac{R i R e^{2}}{8} G_{1}(R i R e)-R e G_{2}(R i R e)$

It can be noticed that the Reynolds number of the upward flow is expressed only as a function of the Richardson and Reynolds numbers associated with the global flow, and there is no need to use the Grashof number. Of course, it is true that $G r=$ $R i R e^{2}$, but there is no logical or physical reason to introduce $G r$ in the expression of eq. (62), given in addition the fact that $G r$ is not a similarity criterion in mixed convection: Ri and Re are then sufficient to completely describe the whole convective domain.

Two possible situations are now examined.

- No reverse flow (RiRe < 288):

$y_{i}=e ; \quad y_{i}^{+}=1 ; \quad G_{1}=0 ; \quad G_{2}=-1 ;$

resulting $R e_{\uparrow} \equiv R e$

The upward flow Reynolds number is identical to the classical one.

\section{- Pure NC:}

$y_{\text {in }}=\frac{e}{2} ; y_{i n}^{+}=\frac{1}{2} ; \quad G_{1 n}=\frac{1}{192}$ and $G_{2}=0$, resulting:

$$
R e_{n \uparrow}=\frac{R i R e^{2}}{8 \times 192}=6.5110^{-4} R i R e^{2}
$$

Equations (57b) and (58) allow, for the upward flow, the computation of velocity and flow rate:

$$
V_{m, n \uparrow}=\frac{g \beta \Delta T e^{2}}{192 v} ; \quad q_{v, n \uparrow}=\frac{g \beta \Delta T e^{3}}{384 v}
$$

And finally, the buoyancy ratio of the upward flow has the value, eq. (59):

$$
(\text { Ri Re })_{n \uparrow}=192
$$

The conclusions for the bidirectional approach are the following:

- from $F C$ to $N C$, the parameters of the upward flow keep finite values;

- a turning point still exists in the description for $\operatorname{RiRe}=288$; 
- the Grashof number is not needed, even in the pure NC.

It is noteworthy that analogue results are obtained when considering the friction factor.

\section{Quadratic approach}

Another way of avoiding the difficulties related to the definition of $V_{m}$ is to consider square mean values. The velocity $\sqrt{U^{2}}$ is then used, with:

$$
\overline{U^{2}}=\frac{1}{e} \int_{0}^{e} U^{2} d y
$$

It is noted then that the bulk velocity $V_{m}$ is based on the mass flow rate, while the square mean velocity is based on the kinetic energy.

By using the local velocity decomposition (20), eq. (65a) becomes:

$$
\overline{U^{2}}=\frac{1}{e} \int_{0}^{e}\left(U_{n}^{2}+U_{f}^{2}+2 U_{n} U_{f}\right) d y
$$

The result can be summerized as:

$$
\begin{aligned}
\overline{U^{2}} & =\text { terms in }\left(\frac{\rho g \beta \Delta T e^{2}}{\mu}\right)^{2} \\
& + \text { terms in } V_{m}^{2}+\text { terms in } \frac{\rho g \beta \Delta T e^{2} V_{m}}{\mu}
\end{aligned}
$$

This allows the definition of a quadratic Reynolds number $\operatorname{Re}_{Q}$ :

$$
\operatorname{Re}_{Q}^{2}=\frac{\overline{U^{2}}(2 e)^{2}}{v^{2}}
$$

Which is of the form:

$\operatorname{Re}_{Q}^{2}=$ terms in $R i^{2} \mathrm{Re}^{4}+$ terms in $\mathrm{Re}^{2}$

+ terms in $\operatorname{RiRe}^{3}$

The turning point for RiRe $=288$ does not occur here and the Grashof number is still absent.

\section{- In pure FC:}

$R i=0$ and $U \equiv U_{f}$, resulting:

$$
\sqrt{\overline{U_{f}^{2}}}=1.095 V_{m} ; \quad R e_{Q f}=1.095 R e
$$

The new quadratic Reynolds number does not coincide with the classical one. It is superior by about $10 \%$.

\section{- In pure NC:}

In that case: $\operatorname{Re}=0$ and $U=U_{n}$, resulting:

$$
R e_{Q n}=1.44 \cdot 10^{-4} \operatorname{RiRe}^{2} \neq 0
$$

The conclusions for the quadratic approach are:

- from FC to NC, the quadratic parameters keep finite values;

- there is a perfect continuity in describing FC-MC-NC;

- the Grashof number is not needed in the description.

\section{Numerical example}

Coming back to an isothermal flow, $R e=1412$ corresponds to a flow rate of $q_{v}=6.10^{-4} \mathrm{~m}^{3} / \mathrm{s}$ (for a width $l=1 \mathrm{~m}$ ).

In pure $\mathrm{NC}\left(V_{m}=0\right)$, the same Reynolds number in the upward flow $\left(R e_{n \uparrow}=1412\right)$ can be obtained with a temperature gap of $\Delta T=6.26^{\circ} \mathrm{C}$, resulting in $R i=1.083$.

Then $V_{d n \uparrow}=6.10^{-2} \mathrm{~m} / \mathrm{s} ; q_{v}=6.10^{-4} \mathrm{~m}^{3} / \mathrm{s}$ : the velocity is two times the velocity of the isothermal flow, but as the cross section is divided by $2(e / 2$ instead of $e$ ), the flow rate is not changed.

As for the quadratic approach, it leads to:

$$
\sqrt{\overline{U_{n}^{2}}}=6.6 \cdot 10^{-2} \mathrm{~m} / \mathrm{s} ; \operatorname{Re}_{Q n}=311
$$

It can be observed that the ratio "quadratic velocity" over $V_{d n \uparrow}$ is almost the same as in an isothermal flow (eq. 67).

\section{The bulk temperature}

The bulk temperature definition presented in eq. (55) may be adapted to both the bidirectional and quadratic approaches.

* The upward flow bulk temperature, as defined over the section $\left[0, y_{i}\right]$, becomes: 


$$
T_{m \uparrow}=\frac{1}{V_{m \uparrow} y_{i}} \int_{0}^{y_{i}} T U d y
$$

If $\operatorname{RiRe}<288$ (no reverse flow, $y_{i}=e, V_{m \uparrow} \equiv V_{m}$ ), then $T_{m \uparrow} \equiv T_{m}$.

- The mean fluid temperature relative to the square mean velocity, is given by:

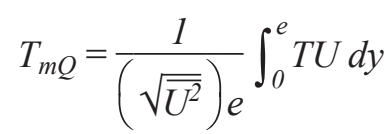

In particular, in forced convection, $T_{m}$ is modified in the same proportion as Reynolds number, eq. (67):

$$
T_{m Q f}=\frac{1}{1.095} T_{m}
$$

The analytical expressions of $T_{m \uparrow}$ and $T_{m Q}$ are rather complex, but it is sufficient to indicate here that the mean fluid temperature has finite values in both situations. From the point of view of the raised question, they are thus equally satisfactory.

\section{What approach to recommend?}

A first general remark is that, in both approaches, a unifying formulation has been achieved for the entire convection domain and that this formulation includes only the Richardson and Reynods numbers.

The summary of all advantages and disadvantages of each method follows.

\section{* The bidirectional approach}

- takes away the difficulties related to the definitions of the mean velocity $V_{m}$ and of the mean fluid temperature $T_{m}$;

- allows the distinction between uni- and bidirectional flow;

- does not imply any modification of usages for the uni-directional flow;

- is adaptable to boundary layer flows.

\section{But}

- it introduces a turning point between the uni- and bi-directional flow;

- it supposes the knowledge of $y_{i}\left(\right.$ where $\left.U\left(y_{i}\right)=0\right)$.

- The quadratic approach

- also takes away the difficulties posed by $V_{m}$ and $T_{m}$;

- is also adaptable to the boundary layer flows.

\section{But}

- it is global, the uni- and bidirectional flows are no longer differentiated;

- it implies a significant modification of the current usages, $V_{m}$ and $R e$ being replaced by $\sqrt{\overline{U^{2}}}$ and $R e_{Q}$ respectively.

The above comparison seems to give some advantage to the bidirectional approach, which is more physical and more compatible with the usual formulations.

\section{Expression of the heat transfer}

The fully developed flow used as example in this study is not the most appropriate to study the thermal aspect of the problem, given the fact that the temperature field is purely conductive. In other configurations, selection between natural convection and forced convection has been based on Stanton (or Nusselt) number (Kakaç and Yener 1995, Taine and Petit 1989), which is not possible here: the flux density at the wall is given by $\varphi_{p}=$ $\lambda \Delta T / e$ and, the hydraulic diameter being equal to $2 e$, the Stanton number becomes:

$$
S t=\frac{\varphi_{p}}{\rho C_{p} V_{m} \Delta T}=\frac{2}{R e P r}
$$

Certainly, when using the bidirectional approach, it can be defined an upward flow Stanton number, like for the Reynolds number:

$$
S t_{\uparrow}=\frac{2}{R e_{\uparrow} P r}
$$


But in the present case, with a definition or another, the Stanton number has no property in the selection of the convective regime; and Nusselt number, a constant $(N u=2)$, even less.

\section{CONCLUSIONS}

This article is aimed at underlining three difficulties encountered in convective heat transfer and to offer some answers.

The first difficulty represents a preambule: it concerns the expression of the reference temperature used in the Boussinesq approximation, which must not be arbitrarily chosen. In particular, in the case of a thermally and hydraulically fully developed fluid flow, a physical reasoning led to the conclusion that the reference temperature must be the average temperature field in a horizontal crosssection of the flow.

A second question, of practical nature, was raised in connection to the "borders" of the three convective domains (FC, MC and $\mathrm{NC}$ ), and to the possible criteria to distinguish among them. Different criteria have been proposed based on existing forces, on mechanical energy, on dissipation and on entropy production. With the exception of the last one, all had in common the sole dependency on the buoyancy coefficient $R i \cdot R e$ and all had comparable orders of magnitude. A synthesis of the results showed that a practical range for the mixed convection is from $R i \cdot R e=50$ to $R i \cdot R e=2000$. Below 50, buoyancy is negligible (dominant FC), and over 2000, the pressure gradient is negligible (dominant $\mathrm{NC}$ ). Nevertheless, the presented values were obtained for a particular situation and must be used with precaution for other physical configurations.

The third problem, more fundamental, aims to unify the FC-MC-NC description in the similarity framework, in order to ensure the physical continuity of the dimensionless governing equations. Two approaches have been proposed, both based on the redefinition of the flow mean velocity: a bidirectional approach which distinguishes between the upward flow and the downward flow when a reverse flow occurs, and another based on the square mean in the whole flow field. In both methods, the continuity of description is well done and the similarity criteria $R i$ and $R e$ are sufficient to describe the totality of the convective domain, without recalling the Grashof number. However, the bidirectional approach is privileged by its surplus of physical transparence and closeness to the current usage.

\section{NOMENCLATURE}

$\overline{a^{2}}, \overline{b^{2}}, \overline{c^{2}}$ mean squares of buoyancy, pressure and viscous terms

e distance between walls, $m$

$\mathrm{Eu} \quad$ Euler number (eq. 10b, 40b)

$g \quad$ gravity acceleration, $m \cdot s^{-2}$

$p^{*} \quad$ pressure, $\mathrm{Pa}$

$q_{v} \quad$ volumetric flow rate, $m^{3} \cdot s^{-1}$

$R i \quad$ Richardson number (eq. 10b, 25c)

Re Reynolds number

Ri Re buoyancy ratio (eq. 25e)

$T$ temperature, ${ }^{\circ} \mathrm{C}$

$T_{1}, T_{2} \quad$ hot wall / cold wall temperature, ${ }^{\circ} \mathrm{C}$

$T \quad=T_{1}-T_{2}$

$U \quad$ fluid velocity component in $x$ direction, $m \cdot s^{-1}$

$V_{m} \quad$ bulk (mean) velocity, $m . s^{-1}$

$x \quad$ vertical coordinate, $m$

$y \quad$ horizontal coordinate, $m$

$\beta \quad$ thermal expansion coefficient of fluid, $K^{-1}$

$\Phi^{\prime \prime \prime} \quad$ local viscous dissipation, W. m $^{-3}$

$\Phi^{\prime \prime} \quad$ viscous dissipation on a plane section, W. $m^{-2}$

$\mu \quad$ dynamic viscosity of fluid, $\mathrm{kg} \cdot \mathrm{m}^{-1} \cdot \mathrm{s}^{-1}$

$v \quad$ kinematic viscosity of fluid, $m^{2} \cdot s^{-1}$

$\rho \quad$ fluid density, $\mathrm{kg} \cdot \mathrm{m}^{-3}$

$\sigma^{\prime \prime} \quad$ entropy production on a plane section, $W \cdot m^{-2} \cdot K^{-1}$

dimensionless parameter 


\section{RESUMO}

Esse artigo objetiva renovar o interesse na pesquisa em convecção térmica mista e enfatizar três temas que surgem da presente análise: (i) uma clara definição da temperatura de referência na aproximação de Boussinesq; (ii) uma delimitação prática dos tres modos convectivos, que são a convecção forçada (CF), convecção mista (CM) e convecção natural (ou livre) $(\mathrm{CN})$. (iii) e, finalmente, uma descrição uniforme do conjunto $\mathrm{CF} / \mathrm{CM} / \mathrm{CN}$ no contexto de similaridade. O caso plano, para o qual soluções analíticas estão disponíveis, permite uma ilustração detalhada das respostas aqui avançadas para os temas acima.

Palavras-chave: convecção mista, aproximação de Boussinesq, similaridade, forças de empuxo, produção de entropia, escoamento reverso.

\section{REFERENCES}

AUNG W AND WORKU G. 1986. Theory of fully developed combined convection including flow reversal. J Heat Transfer 108: 485-488.

BARLETTA A. 1998. Laminar mixed convection with viscous dissipation in a vertical channel. Int J Heat Mass Transfer 41: 3501-3513.

BARLETTA A AND ZANCHINI E. 1999. On the choice of the reference temperature for fully developed mixed convection in a vertical channel. Int J Heat Mass Transfer 42: 3169-3181.

BAYAZITOGLU Y, PASLAY PR AND CERNOCKY P. 2007. Laminar Bingham fluid flow between vertical parallel plates. Int J Thermal Sci 46: 349-357.

BEJAN A. 1994. Entropy generation through heat and fluid flow, J Wiley \& Sons.

BEJAN A. 1996. Entropy generation mini-mization, CRC Press. BEJAN A. 2004. Convection heat transfer, $3^{\text {rd }}$ ed., J Wiley \& Sons.

Ben Mansour R, Galanis N AND Nguyen CT. 2005. Production d'entropie en convection mixte. Congrès Français de Thermique SFT 05, Reims, France, Actes, p. 179-184.

BOULAMA K AND GALANIS N. 2004. Analytical solution for fully developed mixed convection between parallel vertical plates with heat and mass transfer. J Heat Transfer 126: 381-388.

DESRAYAUD G AND LAURIAT G. 2009. Flow reversal of laminar mixed convection in the entry region of symmetrically heated, vertical plane channel. Int J Thermal Sci 48: 2036-2045.

GebHaRt B, JALURIA Y, MAHAJAN R AND SAMmaKiA B. 1988. Buoyancy-induced flows and transport, Springer.

Glansdorf P AND PRIGogine I. 1971. Structure, stabilité, fluctuations. Masson.
GuYON E, Hulin JP AND Petit L. 1991. Hydrodynamique physique. Inter-Editions, CNRS, Paris.

HERPE J. 2007. Caractérisation des performances des surfaces d'échange basée sur l'évaluation numérique du taux de production d'entropie. Thèse de doctorat, Univ. de Valenciennes, France.

Herpe J, RusseIL S AND BougEARD D. 2007. Numerical analysis of louvered fin heat exchangers. $6^{\text {th }}$ Int. Conf. on Enhanced, compact and ultra-compact heat exchangers. Comm. CHE2007-0021, Postdam, Germany, p. 149-154.

INCROPERA FP AND DE WITT DP. 1985. Fundamentals of heat and mass transfer, J Wiley \& Sons.

Jeng YN, Chen JL AND Aung W. 1992. On the Reynoldsnumber independence of mixed convection in a vertical channel subjected to asymmetric wall temperatures with and without flow reversal. Int J Heat Fluid Flow 13(4): 329-339.

KAKAÇ S AND YENER Y. 1995. Convective heat transfer, CRC Press.

KaVIANY M. 1994. Principles of convective heat transfer, Springer-Verlag.

Kholai O, Boudebous S, Nemouchi Z AND Rebay M. 2010 Pitchwork bifurcation of the mixed convection in a vertical channel. Heat Transfer Research 41(3): 313-323.

Mladin EC, LAChI M, Rebay M AND PADET J. 2006. The entropy generation in transient thermal convection over a finite thickness plate. Congrès COFRET 06, Timisoara, Romania.

PADET J. 2010. Principes des transferts convectifs. $2^{\circ}$ ed., SFT, Ouvrages, www.sft.asso.fr.

PADET J. 2011. Fluides en écoulement; méthodes et modèles. $2^{\circ}$ ed., SFT, www.sft.asso.fr, Ouvrages.

PADET J. 2013. Ecoulements externes gravitaires. SFT, www. sft.asso.fr, Ouvrages.

PADET J, COTTA RM, CHERECHES NC AND EL WAKIL N. 2004 Internal mixed convection: Criteria for transition from natural to forced convection. Conf. ENCIT 2004, Rio de Janeiro, Brazil, Ref. CIT04-0841, 10 p.

PADET J, COTTA RM, CHERECHES NC AND EL WAKIL N. 2005. Convection laminaire interne: critères de sélection pour distinguer les régimes de convection naturelle, mixte ou forcée. Congrès Français de Thermique SFT 05, Reims, France, Actes, p. 209-214.

Padet C, Mladin EC, PADET J AND Dobrovicescu A. 2009 Minimization of entropy production in fully developed mixed thermal convection. Int. ICHMT Symp. on Convective Heat and Mass Transfer CONV 09, comm. $\mathrm{N}^{\circ}$ 69, Hammamet, Tunisia.

RUBIN H AND ATKINSON J. 2001. Environmental fluid mechanics. CRC Press.

STANCIU D, LACHI M, PADET J, DobROVICESCU A AND STOIAN M. 2005. a) Modélisation des irréversibilités volumiques de la convection forcée turbulente. Congrès Français de Thermique SFT 2005, Reims, France, Actes, p. 227-232b) Etude numérique des irréversibilités dans la convection forcée autour d'un réseau de tubes cylindriques, Actes, p. 233-238. 
SutTON J. 1953. Micrometeorology. McGraw-Hill.

TAINE J AND PETIT JP. 1989. Transferts thermiques. Mécanique des fluides anisothermes. Dunod.

TIMSHENKO V. 2010. Eddie Leonardi memorial lecture "Natural convection from earth to space". $14^{\text {th }}$ Int. Heat Transfer Conf., lect. 23354, Washington DC, USA.

WANG Q, Yoo Y and Jaluria Y. 2003. Convection in a horizontal rectangular duct under constant and variable property formulations. Int J Heat Mass Transfer 46: 297-310.
ZANCHINI E. 1998. Effect of viscous dissipation on mixed convection in a vertical channel with boundary conditions of the third kind. Int J Heat Mass Transfer 41: 3949-3959. 
